\title{
A Diagnostic Challenge: Peritoneal Tuberculosis Mimicking Ovarian Cancer
}

\author{
Eva Rulova*, Elizabete Pumpure*, Zenons Romanovskis***, Liga Puksta-Gulbe*, **, *** \\ * Riga Stradins University, Latvia \\ ** Riga East Clinical University Hospital Gailezers, Latvia \\ *** Oncology Centre of Latvia, Latvia
}

\section{Summary}

A 26 years old patient was admitted to hospital with complaints of abdominal pain and febrile temperature. Initially, elevated serum antigen CA-125, ascites and multiple suspicious nodules in the lungs and abdominal cavity on CT were found, thus, diagnosing ovarian cancer. However, after further preoperative examination, the diagnosis of infiltrative pulmonary tuberculosis with extrapulmonary involvement was set, thereby cancelling the surgery. The patient was treated with first-line antituberculosis agents and discharged home. Almost in all other cases, peritoneal tuberculosis is diagnosed only after surgical intervention.

Key words: ascites, ovarian cancer, peritoneal tuberculosis, CA125

\section{AIM OF THE DEMONSTRATION}

Peritoneal tuberculosis may have a similar clinical presentation as ovarian cancer, and laparotomy is generally mandatory to differentiate these two entities in most cases (5). A high index of suspicion is an important factor in early diagnosis, especially in endemic areas. By this case the authors want to

\section{CASE REPORT}

A 26 years old female with severe diffuse abdominal pain and febrile body temperature was admitted to the emergency department. The patient had complaints of abdominal pain for one week, but she denied such symptoms as nausea, vomiting or chills. During ultrasound examination, ascites and elongated wavy shaped, fluid-containing formation with thickened walls on the right adnexa were found, and the patient was hospitalised to the Department of Gynaecology with an unclear diagnosis. Two days later abdominal computed tomography (CT) (Figurel)was performed which led to think about peritoneal carcinomatosis with secondary ascites (Figure 1), and, sequentially, the patient was stationed to the Department of Oncogynaecology for further investigation.

Many punctures of the pouch of Douglas were performed to investigate ascitic fluid content cytologically, but malignancy was not found. On the $8^{\text {th }}$ hospitalization day a blood test showed elevated serum markers: antigen CA-125 (999.8 U/ml) and human epididymis secretory protein 4 (HE4) (71.6 pmol/l) which usually suggests a malignant process in ovaries if patient has above mentioned clinical presentation.

A suspicion of ovarian tumour diagnosis strengthened when a chest X-rays was performed (FigurePicture 22) before the planned operation and led to think about multiple metastasis bilaterally in upper lobes of lungs. Three days later a chest CT was conducted to clarify diagnose; there were foci in upper lobes of lungs which are the characteristic of specific tubercular process.
For further investigation the patient was directed to the Centre of Tuberculosis and Lung Diseases for additional tests because sputum analysis did not reveal mycobacteria.

On the $32^{\text {th }}$ hospitalization day, during fibrobronchoscopy, bronchial washings were taken and consecutively tested on tuberculosis agent. Culture plating, differential testing on BACTEC confirmed the presence of M. tuberculosis in sample. The idIdentification results of $M$. tuberculosis complex with MPT64 Ag test werewas positive; oncologic pathology in histopathological investigation was not found. As a result, definitive diagnose was formed - infiltrative pulmonary tuberculosis with extrapulmonary peritoneal involvement. As $M$. tuberculosis was sensitive to first-line antituberculosis agents, itpacient received a treatment based on the 4-drug regimen: isoniazid, rifampin, pyrazinamide, and ethambutol.

\section{DISCUSSION}

According to European and Latvian Centres for Disease Prevention and Control data, incidence of tuberculosis was 31.3 per 100.000 persons in 2015; the disease is endemic in Latvia.

A possible mechanism to explain the pathogenesis of $\mathrm{PT}$ is the reactivation of latent tuberculous foci in the peritoneum or haematogenous spread from primary pulmonary tuberculosis (5).

Peritoneal tuberculosis in female patients can manifest with many unspecific clinical symptoms and signs which can mimic ovarian cancer, such as pelvic pain, abdominal fullness, elevation levels of serum CA-125, ascites, and/or an adnexal mass. Peritoneal tuberculosis most often reveals in patients 20 - 40 years of age, but ovarian cancer is rare before the age of 40 and reaches a maximum at age 65-75 (1).

The combination of HE4 and CA125 may be recommended as potential biomarkers in the preliminary differential diagnosis of peritoneal tuberculosis from 
epithelial ovarian cancer before the "golden standard" of pathologic diagnosis finally obtained (8). High levels of CA-125 have been reported in patients with pulmonary and extra-pulmonary tuberculosis, including pleural, peritoneal, pelvic, miliary, and intraabdominal disease (3).

PT and peritoneal carcinomatosis are two of the most common causes of exudative ascites. Differentiating between the two disease entities is difficult and it would be helpful to detect ascites adenosine deaminase (ADA) in this case because it has high sensitivity and specificity in the early diagnosis of peritoneal tuberculosis. Ascites ADA activity are approximately five times higher in the cases of PT comparing with peritoneal carcinomatosis. (4) Unfortunately, until now, in described peritoneal tuberculosis cases the diagnosis is determined by the histological analysis of an operation material.

This article emphasizes that diagnose can be detected preoperatively like in this case. Besides, it is important to avoid hasty and irreversible decisions during surgery, for example, ovarectomy which is recommended in case of ovarian cancer. It should be considered that PT is a highly curable disease with antituberculosis agents. (7).

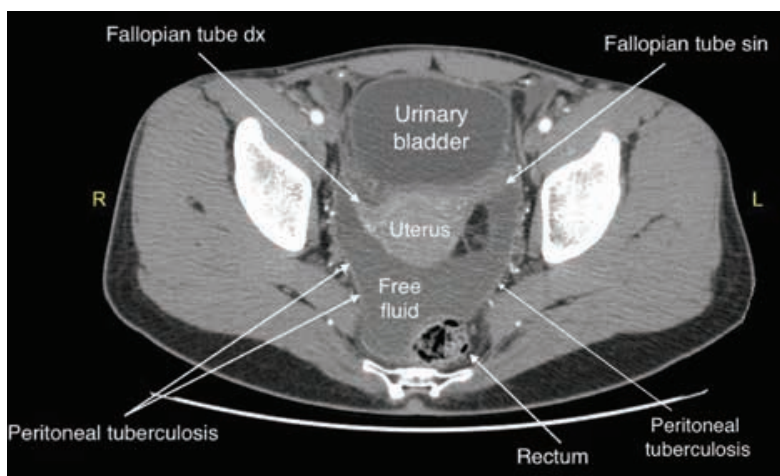

Fig. 1. Peritoneal tuberculosis mimicking peritoneal carcinomatosis with secondary ascites on abdominal and pelvical CT

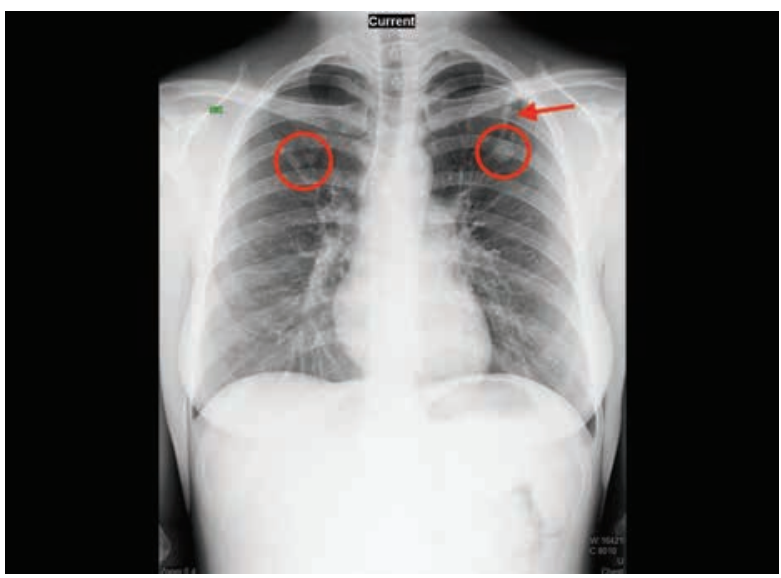

Fig. 2. Foci of tuberculosis mimicking multiple metastases in upper lobes of lungs on a chest $\mathrm{X}$-rays. AP view

\section{Conflict of interest: None}

\section{REFERENCES}

1. Chen-Hsuan Wu, Chan-Chao Chang Chien, ChihWen Tseng, Hung-Yaw Chang, Yu-Che Ou, Hao Lin. Disseminated peritoneal tuberculosis simulating advanced ovarian cancer: A retrospective study of 17 cases // Taiwanese Journal of Obstetrics and Gynecology // 2011; 292-296

2. Debi U, Ravisankar V, Prasad KK, Sinha SK, Sharma AK. Abdominal tuberculosis of the gastrointestinal tract // World Journal of Gastroenterology // 2014; 20(40):14831-14840

3. G.A. Belletti, A.E. Millán, A. López, et al. Pelvic tumor, elevated CA 125 level and tuberculosis // Medicina (B Aires) // 2005, 65 (2) 181-182

4. Kang SJ, Kim JW, Baek JH, et al. Role of ascites adenosine deaminase in differentiating between tuberculous peritonitis and peritoneal carcinomatosis // World Journal of Gastroenterology // 2012; 18(22):2837-2843.

5. Oge T, Ozalp SS, Yalcin OT, Kabukcuoglu S, Kebapci M, Arik D, Isikci T. Peritoneal tuberculosis mimicking ovarian cancer // Eur J Obstet Gynecol Reprod Biol. // 2012; 162(1):105-8

6. S. Koc, G. Beydilli, G. Tulunay, R. Ocalan, N. Boran, N. Ozgul, et al. Peritoneal tuberculosis mimicking advanced ovarian cancer: a retrospective review of 22 cases // Gynecol Oncol // 2006; 565-569

7. T.A. Panoskaltsis, D.A. Moore, D.A. Haidopoulos, A.G. McIndoe. Tuberculous peritonitis; part of the differential diagnosis in ovarian cancer // Am J Obstet Gynecol // 2000; 740-742

8. Zhang L, Chen Y, Liu WX, Wang K. Evaluating the clinical significances of serum HE4 with CA125 in peritoneal tuberculosis and epithelial ovarian cancer // Journal Citation Reports // 2016; 168-172

\section{Address:}

Eva Rulova

Riga Stradins University

Dzirciema street 16, Riga, LV-1007, Latvia.

e-mail: eva.rulova@gmail.com 